\title{
A DNS Study of Self-Accelerating Cylindrical Hydrogen-Air Flames with Detailed Chemistry
}

\author{
Y.X. Xin ${ }^{1}$, C.S. Yoo ${ }^{2,4}$ J.H. Chen ${ }^{2}$ and C. K. Law ${ }^{1,3, *}$ \\ ${ }^{1}$ Department of Mechanical and Aerospace Engineering, \\ Princeton University, Princeton, NJ 08544, USA \\ ${ }^{2}$ Combustion Research Facility, \\ Sandia National Laboratories, Livermore, CA 94551, USA \\ ${ }^{3}$ Center for Combustion Energy, \\ Tsinghua University, Beijing 100084, China \\ ${ }^{4}$ School of Mechanical and Advanced Materials Engineering, \\ Ulsan National Institute of Science and Technology, USN 689-798, Korea
}

* Corresponding Author:

C. K. Law

Department of Mechanical and Aerospace Engineering,

Princeton University, Princeton, NJ 08544, USA

Phone: (609) 258-5271 Fax: (609) 258-6233

Email: cklaw@princeton.edu

Colloquium Topic Area: LAMINAR FLAMES

Total length: 5718

Main text $=3225$ words

Equations $=(1$ lines +2 blank lines $) \times(7.6$ words $/$ line $) \times(1$ column $)=23$ words

References $=(26$ references +2$) \times(2.3$ lines/reference $) \times(7.6$ words/line $)=472$ words

Figure $1=(65 \mathrm{~mm}+10 \mathrm{~mm}) \times(2.2$ words $/ \mathrm{mm}) \times 1$ column $+14=178$ words

Figure $2=(124 \mathrm{~mm}+10 \mathrm{~mm}) \times(2.2$ words $/ \mathrm{mm}) \times 2$ column $+11=602$ words

Figure $3=(53 \mathrm{~mm}+10 \mathrm{~mm}) \times(2.2$ words $/ \mathrm{mm}) \times 1$ column $+21=159$ words

Figure $4=(54 \mathrm{~mm}+10 \mathrm{~mm}) \times(2.2$ words $/ \mathrm{mm}) \times 1$ column $+15=155$ words

Figure $5=(49 \mathrm{~mm}+10 \mathrm{~mm}) \times(2.2$ words $/ \mathrm{mm}) \times 1$ column $+25=154$ words

Figure $6=(53 \mathrm{~mm}+10 \mathrm{~mm}) \times(2.2$ words $/ \mathrm{mm}) \times 1$ column $+12=151$ words

Figure $7=(52 \mathrm{~mm}+10 \mathrm{~mm}) \times(2.2$ words $/ \mathrm{mm}) \times 1$ column $+15=152$ words

Figure $8=(46 \mathrm{~mm}+10 \mathrm{~mm}) \times(2.2$ words $/ \mathrm{mm}) \times 1$ column $+13=136$ words

Figure $9=(51 \mathrm{~mm}+10 \mathrm{~mm}) \times(2.2$ words $/ \mathrm{mm}) \times 1$ column $+10=143$ words

Figure $10=(54 \mathrm{~mm}+10 \mathrm{~mm}) \times(2.2$ words $/ \mathrm{mm}) \times 1$ column $+10=150$ words

Submitted for consideration for presentation at the 35th International Symposium on Combustion and for publication in the Proceedings of the Combustion Institute 


\title{
A DNS Study of Self-Accelerating Cylindrical Hydrogen-Air Flames with Detailed Chemistry
}

\author{
Y.X. Xin ${ }^{1}$, C.S. Yoo ${ }^{2,4}$ J.H. Chen ${ }^{2}$ and C. K. Law ${ }^{1,3, *}$ \\ ${ }^{1}$ Department of Mechanical and Aerospace Engineering, \\ Princeton University, Princeton, NJ 08544, USA \\ ${ }^{2}$ Combustion Research Facility, \\ Sandia National Laboratories, Livermore, CA 94551, USA \\ ${ }^{3}$ Center for Combustion Energy, \\ Tsinghua University, Beijing 100084, China \\ ${ }^{4}$ School of Mechanical and Advanced Materials Engineering, \\ Ulsan National Institute of Science and Technology, USN 689-798, Korea
}

\begin{abstract}
The self-accelerating expanding cylindrical stoichiometric hydrogen-air flames at eight atmospheres were studied via two-dimensional direct numerical simulation (DNS) of the full compressible Navier-Stokes equations with detailed chemistry. The flame morphology and propagation were finely resolved by the application of a time step of $2.5 \mathrm{~ns}$ and a grid size of 4 microns. Temporally, the intermittent propagation of the flame front is captured through examining its propagation velocity. Spatially, the flame front is found to be comprised of segments exhibiting similar propagation properties, i.e. the intermittent instantaneous propagation of the flame front is attributed to the development of cellular structures induced by hydrodynamic instability. The long-term average propagation velocity of the flame front is described by a power law, with a self-acceleration exponent of 1.22 for the flame radius with respect to time. The increase in the global flame velocity is shown to be primarily a consequence of increased flame surface area, with the local front propagation velocity remaining largely at the constant laminar flame speed for the near-unity Lewis number mixture studied herein.
\end{abstract}

Key words: cylindrical flame; hydrogen-air flames; self-acceleration; hydrodynamic instability

* Corresponding author: cklaw@princeton.edu 


\section{Introduction}

The celebrated analyses of Darrieus [1] and Landau [2] demonstrated that the propagation of a premixed flame of infinitesimally thin front is hydrodynamically unstable, characterized by the evolution of cells over the front due to the density jump across it. When the flame is in addition expanding, as in cylindrical and spherical flames, the continuously increasing flame dimension suggests the possible accommodation of progressively more cells. This could then lead to increased total flame surface as compared to that of the otherwise smooth flame, and hence the concomitant increase in the global flame propagation rate, resulting in the so-called self-acceleration of the global flame front. Consequently, if the instantaneous global flame radius is designated by a power law in time $t$ with an exponent $\alpha$, then the flame undergoes self-acceleration if $\alpha>1$. Furthermore, if $\alpha$ is a constant over a period of time, then the propagation is self-similar within this period. It has also been suggested that, for non-integer values of $\alpha$, the wrinkled flame surface and thereby the propagation phenomenon could assume a fractal character. If $\alpha$ in addition assumes the particular value of 1.5 [3], which has been shown to be the fractal dimension of Kolmogorov turbulence [4-5], then it has been further suggested that the flame has undergone self-turbulization, assuming the characteristics of a turbulent flame. This last observation is particularly intriguing, and has generated considerable interest to experimentally or computationally demonstrate that such a value can indeed be attained. A definitive verification however has not been demonstrated.

To substantiate the last statement in terms of computational investigation, which is the present approach, we first note that because of the prohibitive computational cost, previous investigations have invariably adopted the $2 \mathrm{D}$, cylindrical instead of the $3 \mathrm{D}$, spherical configuration. While such an approach is meaningful, some studies using cylindrical flames have however incorrectly claimed the $\alpha$ $=1.5$ value, which is relevant only for spherical flames. As pointed out in [6], this is conceptually 
inappropriate in that a 2-D cylindrical flame intrinsically undergoes less pronounced acceleration than a 3-D spherical flame. Furthermore, most of these studies used the Sivashinsky equation or a modified form of it [7-11], while Ashurst [12] conducted 2-D Lagrangian simulations with a potential flow model, and Aldredge and Zuo [13] used a level-set formulation and Euler equation. Only Liberman et al. [6] performed 2-D simulations by direct numerical simulation (DNS) of the Navier-Stokes equations, with one-step overall chemistry.

The above discussion demonstrates that, in spite of the fundamental and practical importance of the phenomenon of interest, and the fact that the fluid mechanical, transport, and reaction components governing it are well defined, a detailed, rigorous study based on DNS of the fluid flow down to the fine scales needed to resolve the flame structure, together with a detailed reaction mechanism to properly describe the chemistry, has not been performed. Furthermore, there has also been considerable latitude in the extraction of the acceleration exponent in previous studies, which has led to substantial ambiguity in assessing the issues of self-acceleration, self-similarity and self-turbulization mentioned above.

In response to the above considerations, we have performed a DNS study of an expanding cylindrical hydrogen-air flame, with a detailed and largely validated chemistry. The DNS has allowed us to perform a detailed study of the morphology and propagation of the wrinkled flame front, providing insight into the relation between the dynamics of the local flame structure and the expanding global flame. The study unambiguously yields the self-acceleration exponent $\alpha$, allowing further exploration of this phenomenon with confidence.

The paper is organized as follows. In the next section, the numerical method is specified. In Section 3, the instantaneous propagation of the simulated flame front is described and discussed. The 
self-acceleration exponent is extracted by various methods in Section 4 , followed by the conclusions in Section 5 .

\section{Numerical Method}

The study involves the DNS of the propagation of a globally cylindrical flame in a stoichiometric hydrogen-air mixture at eight atmospheres of pressure by using the Sandia S3D code, whose numerical scheme [14] is briefly specified as follows. The compressible Navier-Stokes, species continuity, and total energy equations were temporally integrated by a fourth-order explicit Runge-Kutta method [15]. The solution was spatially discretized using an eighth-order central differencing scheme, with a tenth-order filter to remove spurious high-frequency fluctuations in the solution.

The computational domain is a square box of $5 \mathrm{~cm}$ on each side, which is large enough for the full development of hydrodynamic instability determined from a complementary experimental study [16]. A grid size of 4 microns is applied to resolve the fine structure of the flame front, based on the 1-D unstrained planar stoichiometric hydrogen-air flame at eight atmospheres simulated by the PREMIX code [17]. The chemistry of the system is described by a detailed mechanism developed by Li et al. [18], while the reaction rates, thermodynamic and mixture-averaged transport properties are evaluated by the CHEMKIN and TRANSPORT libraries [19, 20] linked to S3D.

The pressure of the computational domain is set at the constant value of eight atmospheres. A 300 $\mathrm{K}$, stoichiometric hydrogen-air mixture is uniformly distributed across the entire domain initially. The Lewis number of the mixture is near unity [21], and as such, the mixture is largely diffusional neutral such that only hydrodynamic instability is activated. The high system pressure substantially reduces the flame thickness, which promotes the onset of hydrodynamic instability and is also expected to further suppress much of the residual unbalanced diffusional-thermal effects. A Gaussian high 
temperature profile is superposed at the domain center to mimic spark ignition of the mixture. Different peak values of the temperature profile were tested such that the selected one barely initiates flame propagation without affecting its subsequent dynamics. Improved non-reflecting inflow/outflow open boundary conditions were also applied [22]. A time step of $2.5 \mathrm{~ns}$ was adopted, and the solutions of flow velocity, temperature, species mass fraction at each grid were stored every 800 steps, which corresponds to a temporal frequency of $2 \mu \mathrm{s}$. The flame was simulated for $1.7 \mathrm{~ms}$ at which time the flame front reaches the boundary.

\section{Instantaneous Propagation of Flame Front}

For the self-accelerated propagation of spherical flames, experimental measurement typically yields the relationship between the global flame radius and the propagation time, $R(t) \sim t$. However, the focus of the present study is also to determine the evolution of the propagation velocity, $V(t)$. In experimental studies, since the propagation velocity is derived from the flame radius through numerical differentiation, the experimental error is amplified in this process and as such can significantly affect the calculated propagation velocity. In contrast, since the present simulation has extremely fine temporal and spatial resolution, a more reliable propagation velocity can be deduced.

Figure 1 shows the evolution of the simulated flame front at specific time instants, with an interval of $0.2 \mathrm{~ms}$. The flame front is identified as an iso-surface of the progress variable $c=0.5$, where $c=Y_{\mathrm{H}_{2} \mathrm{O}} / Y_{\mathrm{H}_{2} \mathrm{O}}^{E q}$ and $Y_{\mathrm{H}_{2} \mathrm{O}}^{E q}$ is the equilibrium water mass fraction behind the flame front. It is seen that the global flame front exhibits a fractal-like structure: humps and cups of a smaller scale superimposed on those of a larger scale. Thus the otherwise smooth flame front is wrinkled by the hydrodynamic instability, and a cascade of cells continuously develops in the course of the front expansion. That is, when the curvature of the expanding flame becomes large compared to the cutoff length scale, then the 
flame front becomes unstable and breaks up into smaller cells, which subsequently grow until their length scale again becomes large enough to result in a new generation of cells.

We shall discuss the propagation of the flame front from three aspects defining the front: mean radius, propagation velocity and burning intensity. Furthermore, the mean radius of the flame front, $R$, is evaluated by three different methods, as illustrated in Figures $2 \mathrm{a}-2 \mathrm{c}$. Here MD refers to the mean distance of the flame front from the domain center, while AR and CR are derived from the area $A$ enclosed by the flame front, as $\sqrt{A / \pi}$, but with $A$ determined differently. Specifically, for AR, $A$ is simply counted as the number of grids enclosed by the flame front, while for $\mathrm{CR}, A$ is determined from consumption of the fresh mixture. That is, the mass of the consumed fresh mixture at a specific time, $m_{u}^{c}$, is computed through integrating the component change over the entire domain, and accordingly, $A$ is calculated as $m_{u}^{c} / \rho_{b}$ by considering thermal expansion. Figure $2 \mathrm{~d}$ shows that these three methods yield an identical result of $R \sim t$.

The instantaneous (downstream) propagation velocity, $V$, is extracted from the correlation of $R \sim t$ . At a certain time, $R$ is locally fitted by a polynomial with respect to $t$ over a range of $0.1 \mathrm{~ms}$, which analytically yields the instantaneous propagation velocity. Figure 3 presents the instantaneous propagation velocities derived from the three sets of data. The solid line, labeled with "Full set", is based on the simulation results of all $R \sim t$ with the time interval of $2 \mu \mathrm{s}$. The stars, labeled with "Quarter set", adopt only $25 \%$ of the simulation results of $R \sim t$ with the fourfold time interval of $8 \mu$ s. The circle-solid line, labeled with "Half domain", is generated from the simulation conducted in a $2.5 \times 2.5 \mathrm{~cm}^{2}$ domain. For the numerical noise, the stars closely distribute across the solid line, indicating that the recorded $R \sim t$ is dense enough to cancel out the numerical noise within the differentiation process. To understand the influence of the physical boundary, the solid and circle-solid 
line are shown to collapse onto a single curve before $0.7 \mathrm{~ms}$, corresponding to a flame diameter of 1.9 $\mathrm{cm}$, or $75 \%$ of the domain length of $2.5 \mathrm{~cm}$; hence, the regime is free of boundary effects.

It is further seen that the propagation velocity remains constant until $0.3 \mathrm{~ms}$, at which time it quickly increases, and hence indicates the onset of the hydrodynamic instability. Furthermore, the curve displays a wavy shape after $0.8 \mathrm{~ms}$, demonstrating that cell formation leads to intermittent propagation of the global flame front. This feature was reported in previous simulations using the Sivashinsky equation $[7,10]$, but was not observed experimentally due to the much coarser temporal resolution of the experiment. To substantiate this assessment, we have conducted an independent experiment following the same procedure as that of [16], and measured the temporal variation of the mean radius of the expanding spherical flame. The instantaneous propagation velocity is then deduced through the same numerical procedure applied to the DNS results. Figure 4 shows the propagation velocity fitted from the experimental and DNS results with a typical experimental time resolution of $0.03 \mathrm{~ms}$. It is then seen that these two propagation velocities scatter to a similar extent, and the fine-scale waviness is largely suppressed. The $3 \mathrm{D}$ spherical flame propagates faster than the $2 \mathrm{D}$ cylindrical flame due to the larger surface area density caused by the fractal excess. The best estimation is derived from the Sivashinsky equation, suggesting a fractal excess which is two times larger in 3D than in 2D, as studied by Liberman et al. [6]

To understand the globally intermittent nature of the flame propagation, the propagation velocities of different segments of the flame front are spatially and temporally resolved. Every point of the flame front is indexed by its distance $R_{l}$ and azimuthal angle $\theta$, in radians, with respect to the domain center, and the local shape of the flame front is characterized by the correlation of $R_{l} \sim \theta$. The global flame front is divided into in increments of 0.002 azimuthal angle, and the local propagation velocity of the flame front is calculated by the change of the mean radius of each segment with respect to time. Figure 
5 shows the local shape and propagation velocity of the flame front at $1 \mathrm{~ms}$. It is seen that the flame front is composed of various cells, and hydrodynamic instability causes different segments within the same cell to propagate at different velocities, which peak at the center and achieve local minima at the edge. During early stages of cell development, most segments propagate at a velocity close to the center, while the non-uniformity of the velocity distribution becomes most pronounced during the late stage of the cell development. Figure 6 shows the temporal evolution of the local propagation velocity at three different azimuthal angles, demonstrating that their behaviors are consistent with that of the global flame front, and hence implying that the flame front can be treated as an ensemble of flame segments with similar propagation characteristics. This observation is physically reasonable in that the flame front is surrounded by a uniform fresh mixture, and hence different segments would propagate into the same environment and as such should behave similarly. Results from Figures 5 and 6 therefore suggest that the flame front is synthesized from unit cell structures such that pulsation of the global flame is a consequence of the pulsation of the individual segments.

Figure 7 focuses on the propagation of a single segment, which correlates with the instantaneous shape and propagation velocity of a segment with an azimuthal angle of 1.35 radiant. It is seen that pulsation in the propagation velocity coincides with the development of the cell structure. As illustrated in Figure 5, hydrodynamic instability imposes varied influence on the cell structure, accelerating the center but decelerating the edge. For the segment between the center and edge, the net effect depends on the relative distance with respect to the center and edge. At an early stage of cell development, the cell length is small and most segments within the cell structure are located close to the center; whereas at a late stage, the cell length is significantly enlarged, and most segments within the cell structure are distributed closer to the edge rather than the center. In a statistical sense, a segment typically initially accelerates and ends up decelerating. Eventually, when the cell length 
becomes larger than the cut-off length scale of the hydrodynamic instability, fast and slow segments cannot be maintained within the same cell structure. The cell breaks up into a few smaller cells of a new generation, and as such the process repeats anew.

The burning intensity of the flame front can be represented by the mean heat release rate, $\overline{h r_{m}}$, and is plotted in Figure 8 (a), with the values being properly normalized. A 1-D planar stoichiometric hydrogen-air flame at eight atmospheres is simulated by PREMIX, and the heat release rate at the location where the mass fraction of $\mathrm{H}_{2} \mathrm{O}$ equals half of the equilibrium value is used in the normalization. It is seen that after $0.8 \mathrm{~ms}$, the normalized burning intensity varies in a tight range of $1.00 \pm 0.01$. This fact substantiates the assumption that, for the present near-unity Lewis number mixture, the local burning rate is invariant, and hence identical to that of the planar flame, consistent with previous studies, e.g. the work by Bastiaans et al. [23]. Consequently, the self-acceleration behavior is solely caused by the increased surface area density, and therefore is a geometrical property of the flame front. This statement is further scrutinized in Figure 8 (b), in which we have plotted the normalized flame speed,

$$
\bar{V}=\frac{V / F L}{s_{L} / 2 \pi R}
$$

defined as the flame speed $(V)$ divided by the length of the wrinkled surface $(F L)$, normalized by the independently computed laminar flame speed $\left(s_{L}\right)$ divided by the circumference of the global flame $(2 \pi R)$. It is seen that this parameter is almost unity, hence again substantiating the concept that the local burning intensity is not affected by the wrinkling, and that the increase in the global flame speed is simply due to the increase in the flame surface through wrinkling. The slight reduction from unity, by $\sim 2 \%$, is likely caused by residual stretch effects. Indeed, an independent assessment of the mixture 
Lewis number $[24,25]$ yielded a value of 1.1 , which is slightly larger than unity. Since the expanding global flame has a positive curvature, while the local flame fronts also tend to be dominated by positive curvature due to Huygens propagation [26], the net effect of positive curvature and $L e>1$ mixture is a reduction in the propagation velocity. Furthermore, an independent evaluation of the effect of stretch on the expanding flame before the onset of instability, using the expression of [25] with $L e=1.1$ and a global activation energy of $40 \mathrm{kcal} / \mathrm{mol}$ [26], yields a reduction in the flame speed of about $1 \%$, which captures the same range of reduction shown in Figure 8. The relatively small stretch effect, for $L e=1.1$, is amplified by the small flame thickness at the elevated pressure of eight atmospheres which reduces the unbalanced diffusional-thermal effects.

\section{Extraction of Self-Acceleration Exponent}

The above results show that the instantaneous propagation of the flame front is not monotonically accelerating; rather the propagation is intermittent and as such a power-like formula only describes the long term average behavior of the propagation. Consequently, the self-acceleration exponent $\alpha$ needs to be extracted from either the mean radius $R$ or the mean propagation velocity $V$. For the former, $\alpha$ is fitted by the formula $R=R_{0}+\mathrm{A} t^{\alpha}$ as in previous studies [7-13], while for the latter, $\alpha$ is defined by the formula $\ln V=\ln (\mathrm{A} \alpha)+(\alpha-1) \ln (t)$. Mathematically, these two formulas are equivalent but numerically the second correlation could be more reliable because it involves fewer parameters in the fitting. Furthermore, a linear fitting is easier achieved than a nonlinear one. It is then of interest to note from Figures 9 and 10 that nearly identical acceleration exponents of 1.220 and 1.216 are obtained from these distinct fitting formulas over 0.8 to $1.4 \mathrm{~ms}$, for which the hydrodynamic instability is well established and simulation results are not contaminated by boundary effects. This observation therefore confirms that the information contained in the original flame front is well preserved in the 
propagation velocity.

We finally note that in previous studies $[3,6]$, the self-acceleration exponent $\alpha$ is correlated to the fractal excess $d$ by $d=1-1 / \alpha$. Using the self-acceleration exponent 1.22 extracted from our results, a fractal excess $d$ of 0.174 is obtained. Furthermore, assuming the fractal excess of a 3D spherical flame is twice that of a $2 \mathrm{D}$ cylindrical flame, as suggested in [11], then the corresponding acceleration exponent for the spherical flame propagation is 1.54 , which exceeds the self-turbulization threshold of 1.5. Implications of this interesting result await further study, especially considering the approximate nature through which the factor-of-two relation between the $2 \mathrm{D}$ and $3 \mathrm{D}$ fractal excess is suggested [11].

\section{Conclusions}

The self-accelerated propagation of a cylindrical stoichiometric hydrogen-air flame at eight atmospheres is studied by direct numerical simulation of the Navier-Stokes equations with detailed chemistry. The instantaneous propagation is shown to be intermittent and also globally intermittent due to development of the cell structure caused by the hydrodynamic instability. The instantaneous burning intensity is demonstrated to be invariant after the hydrodynamic instability is established, which substantiates the hypothesis that the self-acceleration is solely a consequence of the increased surface area of the flame front due to the hydrodynamic wrinkling. The long-term average flame propagation is described by a power-law dependence, with an exponent of 1.22 extracted from the evolution of the flame radius and propagation velocity. This exponent for the $2 \mathrm{D}$ cylindrical flame propagation implies a corresponding value of 1.54 for the 3D spherical flame propagation. 


\section{Acknowledgements}

The work at Princeton University and Sandia was supported by the Combustion Energy Frontier Research Center, an Energy Frontier Research Center funded by the US Department of Energy, Office of Basic Energy Sciences under Award Number DE-SC0001198. Sandia is a multiprogram laboratory operated by Sandia Corporation, a Lockheed Martin Company, for the United States Department of Energy under contract DE-AC04-94AL85000. The authors thank Fujia Wu of Princeton University for his assistance in acquiring the experimental data reported herein. This research used resources of the National Energy Research Computing Center (NERSC) which is supported by the Office of Science of the US DOE under Contract No. DE-AC03-76SF00098. 


\section{References}

[1] G. Darrieus, “Propagation d'un front de flamme,' presented at Le congress de Mecanique Appliquee, 1945 (unpublished).

[2] L. D. Landau, E. M. Lifshitz, Fluid Mechanics, Pergamon, Oxford, U.K., 1987, p. 486.

[3] Y. A. Gostintsev, A. G. Istratov, Y.V. Shulenin, Comb. Expl. Shock Waves 24 (5) (1988) 563-569.

[4] K. R. Sreenivasan, C. Meneveau, J. Fluid Mech. 173 (1986) 357-386.

[5] K. R. Sreenivasan, R. R. Prasad, Physical D 38 (1-3) (1989) 322-329.

[6] M. A. Liberman, M. F. Ivanov, O. E. Peil, D. M. Valiev, L.-E. Eriksson, Phys. Fluids 16 (7) (2004) 2476-2482.

[7] L. Filyand, G. Sivashinsky, M. Frankel, Physica D 72 (1-2) (1994) 110-118.

[8] Z. Olami, B. Galanti, O. Kupervasser, I. Procaccia, Phys. Rev. E 55 (3) (1997) 2649-2663.

[9] B. Galanti, O. Kupervasser, Z. Olami, I. Procaccia, Phys. Rev. Lett. 80 (11) (1998) 2477-2480.

[10] K. L. Pan, R. Fursenko, Phys. Fluids 20 (9) (2008) 094107.

[11] S. I. Blinnikov, P. V. Sasorov, Phys. Rev. E 53 (5) (1996) 4827-4841.

[12] W. T. Ashurst, Combust. Theory Modelling 1 (4) (1997) 405-428.

[13] R. Aldredge, B. Zuo, Combust. Flame 127 (3) (2001) 2091-2101.

[14] J. H. Chen, A. Choudhary, B. de Supinski, M. DeVries, E. R. Hawkes, S. Klasky, W. K. Liao, K. L. Ma, J. Mellor-Crummey, N. Podhorszki, R. Sankaran, S. Shende, C. S. Yoo, Comput. Sci. Disc. 2 (2009) 015001.

[15] C. A. Kennedy, M. H. Carpenter, Appl. Numer. Math. 14 (4) (1994) 397-433. 
[16] F. J. Wu, G. Jomaas and C. K. Law, Proceedings of Fall Technical Meeting of the Eastern States Section of the Combustion Institute, University of Connecticut, Storrs, U.S, 2011, paper number B-27.

[17] R. J. Kee, J. F. Grcar, M. D. Smooke, J.A. Miller, PREMIX: A Fortran program for modeling steady laminar one-dimensional premixed flames, Report No. SAND85-8240, Sandia National Laboratories, 1985.

[18] J. Li, Z. Zhao, A. Kazakov, M. Chaos, F. L. Dryer, J.J. Scire, Int. J. Chem. Kinet. 39 (3) (2007) 109-136.

[19] R. J. Kee, F. M. Rupley, E. Meeks, J. A. Miller, CHEMKIN-III: A Fortran Chemical Kinetic Package for the Analysis of Gas-phase Chemical and Plasma Kinetics, Report No. SAND96-8216, Sandia National Laboratories, 1996.

[20] R. J. Kee, G. Dixon-Lewis, J. Warnatz, M. E. Coltrin, J.A. Miller, A Fortran Computer Code Package for the Evaluation of Gas-phase Multicomponent Transport Properties, Report No. SAND86-8246, Sandia National Lab, 1986.

[21] C. J. Sun, C. J. Sung, L. He, C. K. Law, Combust. Flame 118 (1-2) (1999) 108-128.

[22] C. S. Yoo, H. G. Im, Combust. Theory Modelling 11 (2) (2007) 259-286.

[23] R. J. M. Bastiaans, J. A. van Oijen, L. P. H. de Goey, LPH, Phys. Fluids 21 (1) (2009) 015105.

[24] G. Joulin, T. Mitani, Combust. Flame 40 (3) (1981) 235-246.

[25] J. K. Bechtold, M. Matalon, Combust. Flame 127 (1-2) (2001) 1906-1913.

[26] C. K. Law, Combustion Physics, Cambridge University Press, New York, U.S., 2006, pp. 234 \& 282. 
Figures

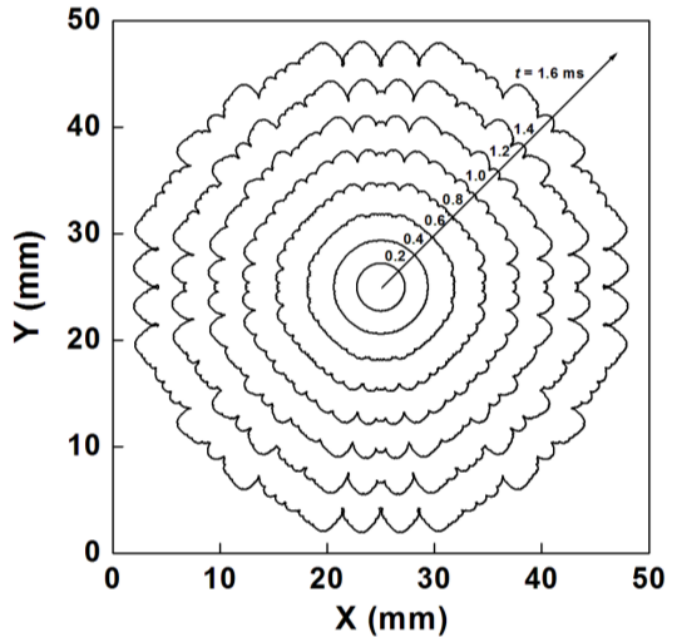

Figure 1. Flame front evolution at selected time instants simulated by direct numerical simulation.

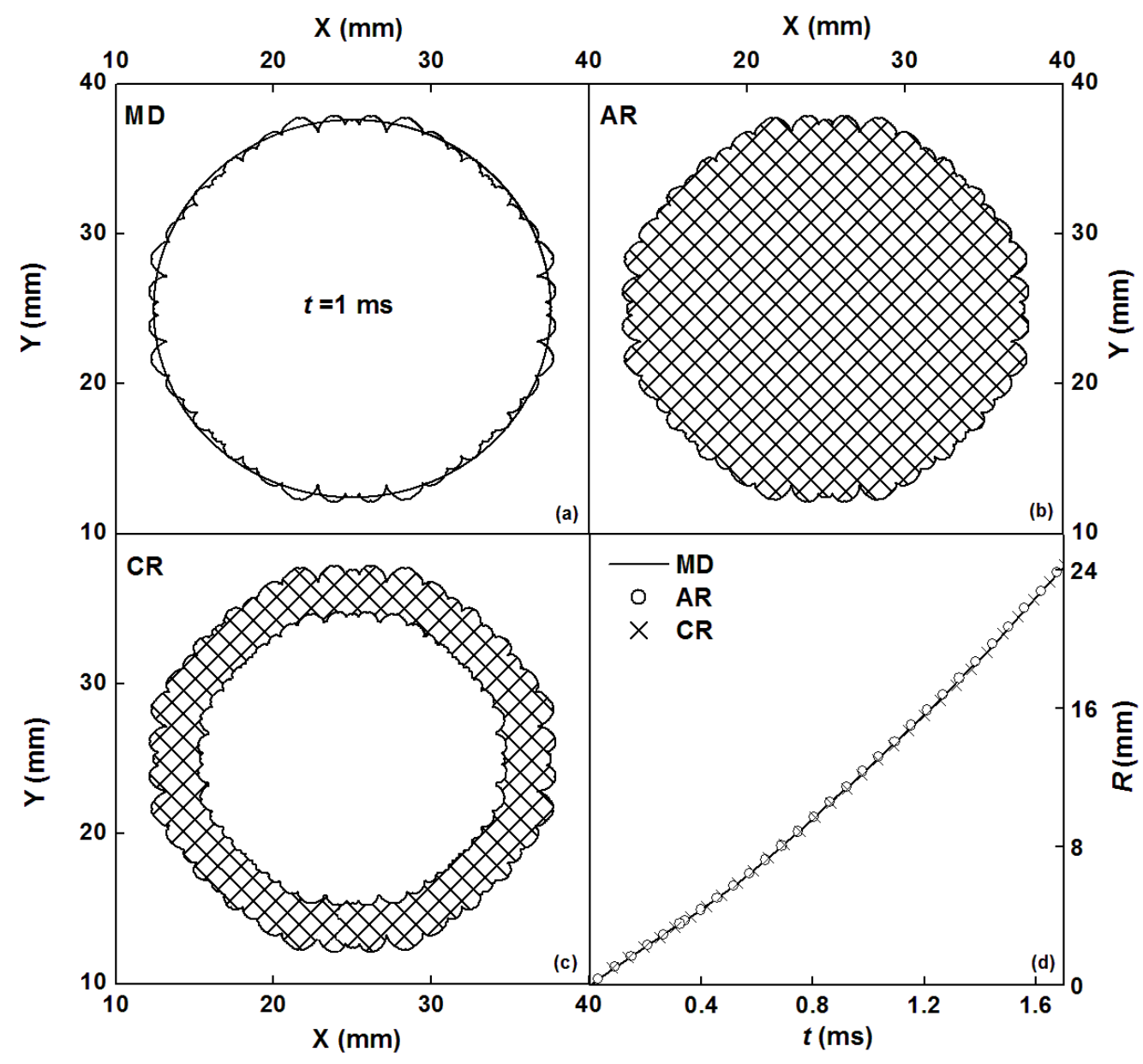

Figure 2. Mean radius of flame front identified by different methods. 


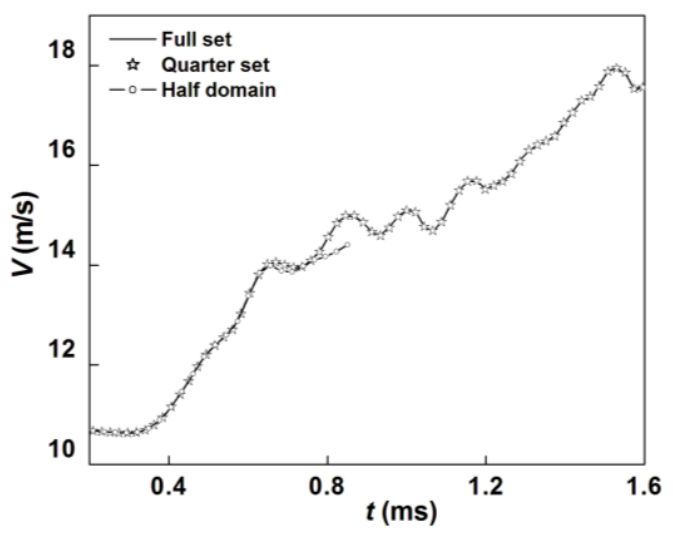

Figure 3. Instantaneous propagation velocity of the flame front fitted from full set, half domain and a quarter set of data.

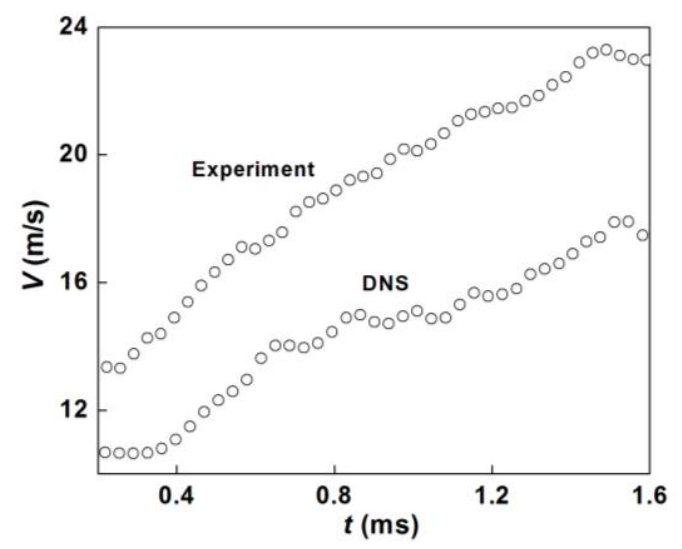

Figure 4. Instantaneous propagation velocity of the flame front fitted from experimental and DNS data.

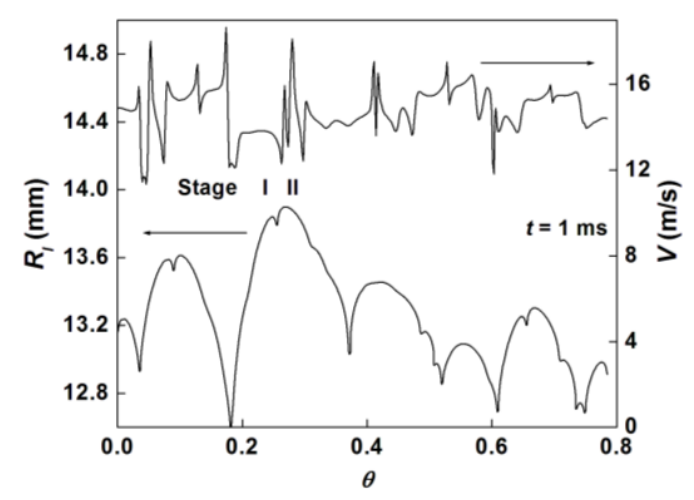

Figure 5. Local shape and propagation velocity of the flame front at $1 \mathrm{~ms}$. Stage I and II: early and late stages of cell development. 


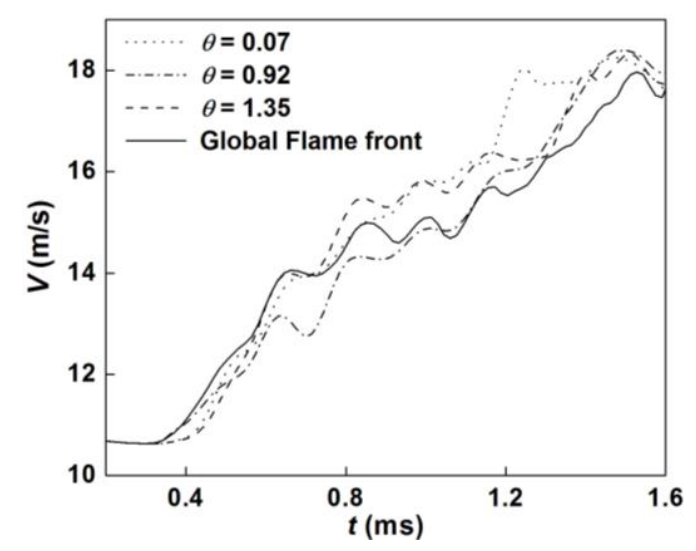

Figure 6. Instantaneous propagation velocity of representative segments of the flame front.

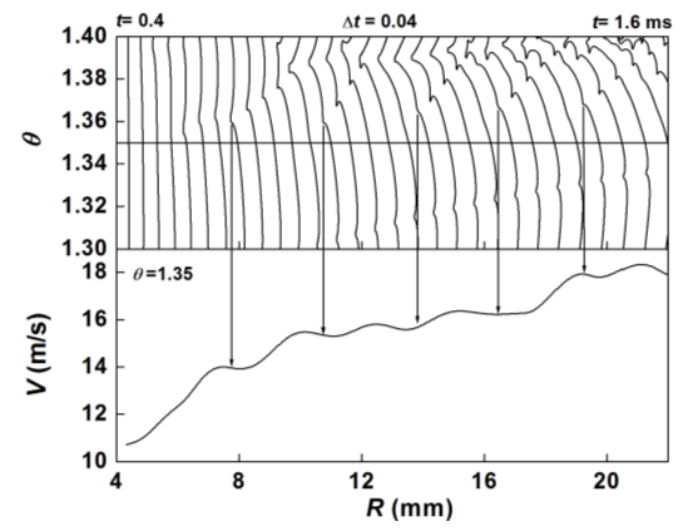

Figure 7. Instantaneous shape and propagation velocity of a representative segment of the flame front.

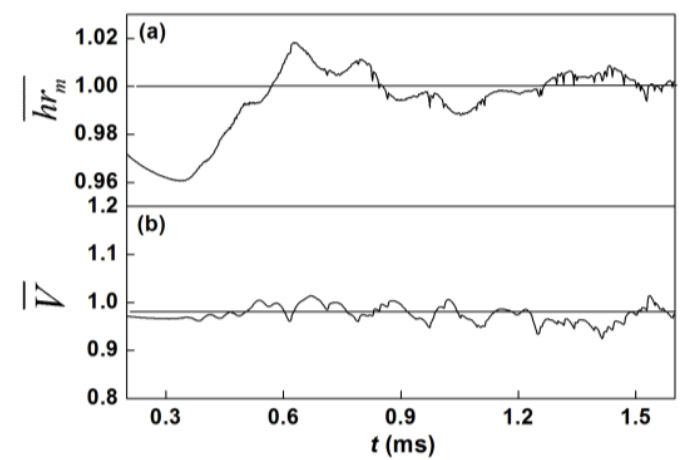

Figure 8. Instantaneous burning intensity and normalized propagation velocity of the flame front. 


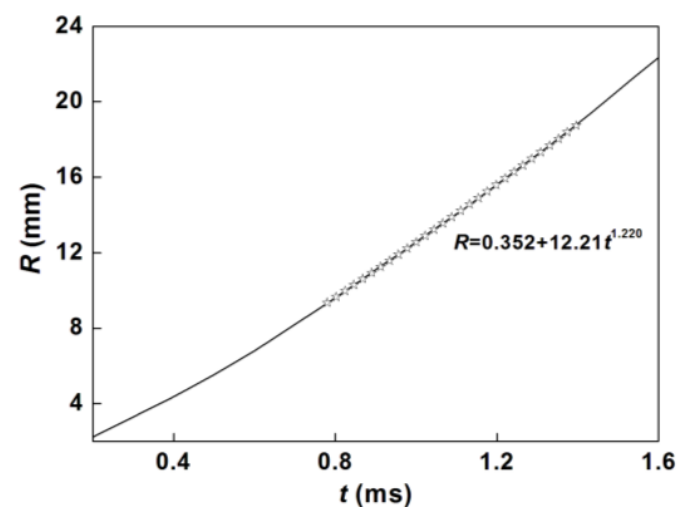

Figure 9. Self-acceleration exponent extracted from the instantaneous mean radius.

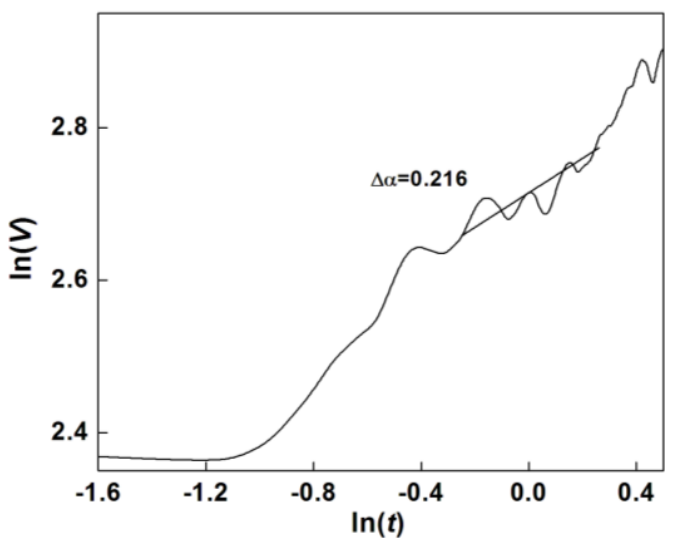

Figure 10. Self-acceleration exponent extracted from the instantaneous propagation velocity. 


\section{Figure Captions}

Figure. 1 Flame front evolution at selected time instants simulated by direct numerical simulation.

Figure. 2 Mean radius of the flame front identified by different methods.

Figure. 3 Instantaneous propagation velocity of the flame front fitted from full set, half domain and a quarter set of data.

Figure. 4 Instantaneous propagation velocity of the flame front fitted from experimental and DNS data.

Figure. 5 Local shape and propagation velocity of the flame front at $1 \mathrm{~ms}$. Stage I and II: early and late stage of cell development.

Figure. 6 Instantaneous propagation velocity of representative segments of the flame front.

Figure. 7 Instantaneous shape and propagation velocity of a representative segment of the flame front.

Figure. 8 Instantaneous burning intensity and normalized propagation velocity of the flame front.

Figure. 9 Self-acceleration exponent extracted from the instantaneous mean flame radius.

Figure. 10 Self-acceleration exponent extracted from the instantaneous propagation velocity. 